\title{
Detection of arcs in Saturn's F ring during the 1995 Sun ring-plane crossing
}

\author{
S. Charnoz ${ }^{1}$, A. Brahic ${ }^{1}$, C. Ferrari ${ }^{1}$, I. Grenier ${ }^{1}$, F. Roddier ${ }^{2}$, and P. Thébault ${ }^{3}$ \\ 1 Équipe Gamma-Gravitation, Service d'Astrophysique, CEA/Saclay, Orme des Merisiers, \\ 91191 Gif-sur-Yvette Cedex, France \\ ${ }^{2}$ Institute for Astronomy, University of Hawai, 2680 Woodlawn Drive, Honolulu, Hawai 96822, Hawai \\ 3 DESPA, Observatoire de Paris, 92195 Meudon Cedex Principal, France
}

Received 24 July 2000 / Accepted 19 October 2000

\begin{abstract}
Observations of the November 1995 Sun crossing of the Saturn's ring-plane made with the $3.6 \mathrm{~m} \mathrm{CFH}$ telescope, using the UHAO adaptive optics system, are presented here. We report the detection of four arcs located in the vicinity of the F ring. They can be seen one day later in HST images. The combination of both data sets gives accurate determinations of their orbits. Semi-major axes range from $140020 \mathrm{~km}$ to $140080 \mathrm{~km}$, with a mean of $140060 \pm 60 \mathrm{~km}$. This is about $150 \mathrm{~km}$ smaller than previous estimates of the $\mathrm{F}$ ring radius from Voyager 1 and 2 data, but close to the orbit of another arc observed at the same epoch in HST images.
\end{abstract}

Key words. planets: individual: Saturn

\section{Introduction}

The Sun's crossing of Saturn's ring-plane is a rare opportunity to observe the unlit face of the rings. Results of observations made at the $3.6 \mathrm{~m} \mathrm{CFH}$ Telescope are reported here. Thanks to the use of the UHAO adaptive optics system (Roddier et al. 2000), high resolution groundbased images were obtained ( $0.15^{\prime \prime}$ in August 1995 and $\sim 0.25^{\prime \prime}$ in November 1995). The Sun's crossing lasted from November 17 th to 21 st 1995 . It was accompanied by three Earth ring plane crossings (1995 May 22nd, August 10th, and 1996 February 11th). Several teams observed these events (Nicholson et al. 1996, hereafter N96; Bosh \& Rivkin 1996; Sicardy et al. 1996; Roddier et al. 1996a; Ferrari et al. 1997).

The unusual viewing of the rings during these events provides a rare opportunity to detect faint structures such as small satellites, clumps, or arcs, that are usually lost in the glare of the bright main rings. In this paper, we focus our attention on the azimuthal structure of the $\mathrm{F}$ ring. Located at the Roche limit of Saturn and bounded by two shepherding satellites Pandora and Prometheus, the $\mathrm{F}$ ring is very intriguing. The Voyager images revealed its complex radial and azimuthal structure including multiple strands, clumps and braids. Interactions with Saturn's

Send offprint requests to: S. Charnoz, e-mail: charnoz@discovery.saclay.cea.fr satellites might explain some features, but they are not completely understood.

During the 1995 ring crossing observing campaign, the F ring appeared populated with numerous features, either point-like or longitudinally extended. The observation of two point-like objects (S/1995 S1 and S/1995 S3) was reported by Bosh \& Rivkin (1996) during the May crossing. During the August crossing, our team discovered at least 6 new features with semi-major axes compatible with the F ring (Roddier et al. 1996a; Ferrari et al. 1997). At least one of them was identified as an arc-like object. During the same period three objects (S/1995 S5, S/19995 S6 and S/1995 S7) were detected by N96. Finally, during the November crossing, two wide $\operatorname{arcs}\left(7^{\circ}\right.$ and $10^{\circ}$-long) were observed by N96. The brightest was also seen by Poulet et al. (2000, hereafter P2000). The most striking aspect of those discoveries is that no evident correlation can be found between features observed in May, August and November (N96, Bosh \& Rivkin 1996, P2000).

In the present paper, we report the detection of 4 arcs in the region of the F ring on November 20th. The same features can be seen in some HST images taken 24 to 36 hours later. These images have been already presented in N96. By combining both sets of data, accurate orbital solutions are derived. The data processing of CFHT and HST images is presented in the first section. Results are presented in the second section and discussed in the final section. 


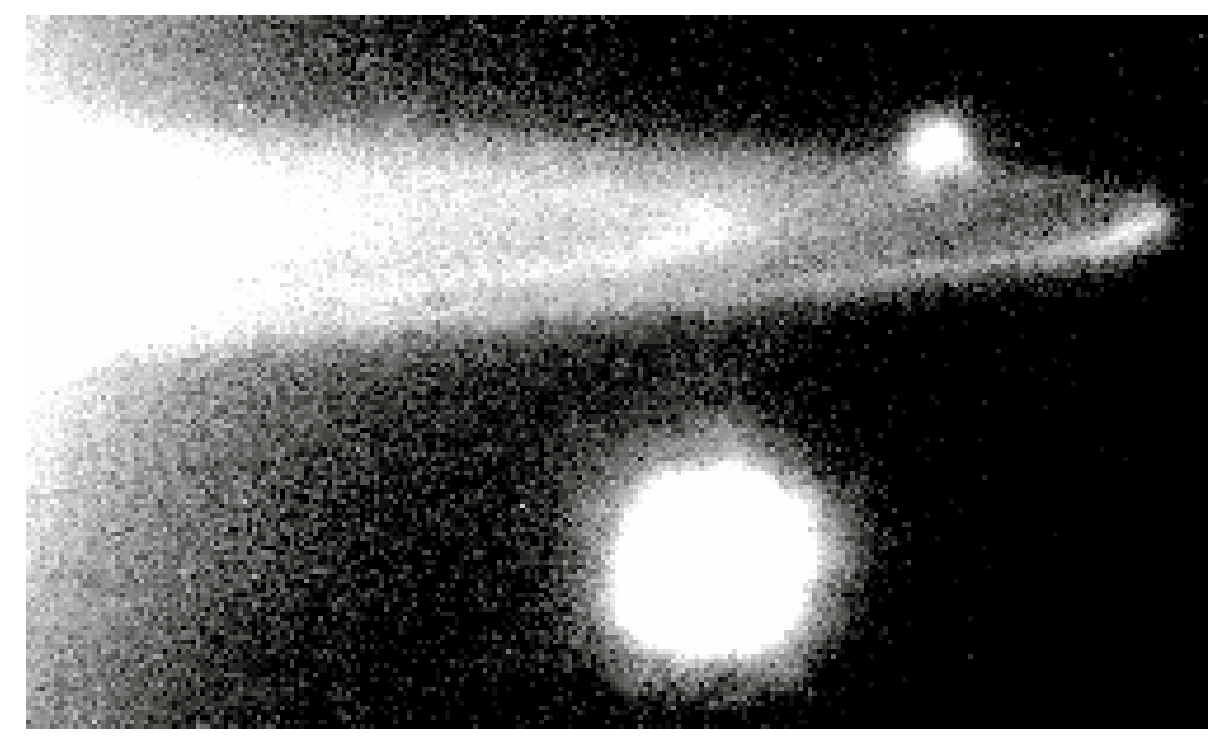

Fig. 1. Image of the West ansa in the $K$ band on 1995 November 21 at 5 h11 UTC, obtained at CFHT with the UHAO system. The F ring and the Cassini division appear brightly. Janus (upper right) and Tethys (bottom center) are visible in this image. The north pole of Saturn is oriented toward the top of the image. This image is slightly saturated as can be seen from the Cassini division and the $\mathrm{F}$ ring

\section{Description of data set and processing}

\subsection{Description of data}

Images were taken at the $3.6 \mathrm{~m} \mathrm{CFH}$ Telescope during four nights from 1995 November 17th to 20th. Three nearinfrared filters $(J, H$ and $K$ ), which correspond to the absorption bands of methane were used to significantly reduce the strong scattered light of Saturn. A $K$ band image is presented in Fig. 1. The best observing conditions were obtained during the last night, the main rings being darkest. During the nights of November 17th, 18th, 19th the stronger brightness of the main rings was not favorable for the detection of dim objects. Consequently only images of November 20th were considered.

At this time the Sun was about $0.01^{\circ}$ below the rings, and the Earth was at $2.67^{\circ}$ above the ring plane, looking at the unlit face. 160 images of the west ansa were obtained from $5 \mathrm{~h}$ to $8 \mathrm{~h} 45$ UT. At this time, there was no satellite on the east ansa bright enough for the adaptive optics tracking system to work properly. In consequence, the CFHT data set contains only images of the west ansa. The resolution has been checked on every image by extracting the point spread function (PSF) of satellites Tethys, Enceladus, Dione and Mimas. The full width at half maximum of the PSF is on average $0.25^{\prime \prime}(1670 \mathrm{~km}$ at Saturn) on the whole data set, ranging from $0.19^{\prime \prime}$ to $0.33^{\prime \prime}$. The size of one pixel in arcsecond is determined by comparing the angular separation of all pair of major satellites (given by the ephemeris) with their separation in pixels as measured on the plates. The result is robust: one pixel subtends $0.035^{\prime \prime}$ ( $\sim 233 \mathrm{~km}$ at Saturn). The exposure times ranged from $15 \mathrm{~s}$ to $30 \mathrm{~s}$.

In order to make a comparative study of two complementary data sets, we have also used the HST images presented in N96, retrieved from the FTP site of the Space Telescope Science Institute. They consist of twenty Planetary Camera images of both ansae (10 images for each ansa), taken with filters centered at $890 \mathrm{~nm}$, between 10h UT and 18h UT on November 21st, at the end of the Sun's crossing. The resolution is $0.1^{\prime \prime}(668 \mathrm{~km}$ at Saturn). The scale of the plates is taken from Holtzman et al. (1995): one pixel subtends $0.045^{\prime \prime}(\sim 304 \mathrm{~km}$ at Saturn). Exposure times range from $300 \mathrm{~s}$ to $500 \mathrm{~s}$. As a consequence, satellites appear smeared due to their keplerian motion.

\subsection{Data processing}

Our data processing has been designed for the detection of faint objects in the $\mathrm{F}$ ring. As a consequence we have not made a photometric study; indeed many images with different exposure times and different filters are combined indiscriminately. A longitudinal profile of the $\mathrm{F}$ ring was built to reveal new objects. To this purpose the following data processing steps were applied to both CFHT and HST images: (i) astrometry, (ii) extraction of the F ring profiles, (iii) subtraction of the F ring background and the correction of geometry and illumination effects.

\subsubsection{Astrometry}

In CFHT images, the center of Saturn and orientation of the planet's North pole axis is determined by a least square fit to the positions of satellites Tethys, Enceladus, Mimas and Dione (using the ephemeris of the Bureau Des Longitudes in Paris). However Mimas and Tethys could not be used in one third of images in which they are observed too close to each other, not suitable for an accurate 
determination of their center. In these images the point of maximum elongation of the Cassini division and of the $\mathrm{F}$ ring were used to better constrain the orientation of Saturn's equatorial axis. The residual on satellites position measurements is $0.09^{\prime \prime}(\sim 2.6$ pixels or $600 \mathrm{~km})$, and reflects a combination of pointing and ephemeris errors.

In HST images, the astrometry has not been done using satellites because of their elongated appearance. The sharp inner edge of the Cassini Division was used instead as a reference. Located at $117577 \mathrm{~km}$ from the center of Saturn, it is known to be slightly eccentric due to the close 2:1 resonance with Mimas. The resulting radial excursion (2ae) is only $150 \mathrm{~km}$, representing 0.5 pixel, or $0.02^{\prime \prime}$ (Porco et al. 1984). The center of Saturn as well as the orientation of the axes are determined by fitting an ellipse with twenty points picked by eye on the reference edge. Corrections of the geometrical distortion for $\mathrm{WF} / \mathrm{PC} 2$ camera (WF/PC2 instrument handbook version 5.0 , provided by the STSCI) is included only when new objects are measured, for the need of their orbit determination. The astrometric error is determined from the residuals of the Cassini division fit. It is $0.035^{\prime \prime}(\sim 0.8$ pixel or $240 \mathrm{~km}$ at Saturn). The correction for geometrical distortion is about 1 pixel, representing less than $0.6^{\circ}$ on the $\mathrm{F}$ ring longitudinal profile, not detectable in Figs. 2 and 3.

\subsubsection{Extraction of the $F$ ring profile}

From $-55^{\circ}$ to $+55^{\circ}$ around maximum elongation, pixel counts are summed perpendicularly to the equator axis, in a box centered on the $\mathrm{F}$ ring (defined as a circle of radius $140200 \mathrm{~km}$ and projected onto the ring plane) with the same width as the PSF. For each image, an intensity profile of the $\mathrm{F}$ ring is obtained as a function of longitude, measured from the point of maximum elongation. The extremity of the ansa (within 5 pixels around maximum elongation) is not considered because the scans become parallel to the ring in this region. The scattered light of Saturn is estimated in the neighborhood of the integration box, outside the F ring, and systematically subtracted. Profiles are then corrected for effects of the different filters and for different exposure times: each profile is multiplied by a normalization factor in order to keep at the same median level of brightness a reference zone of the $\mathrm{F}$ ring, chosen to be between $0^{\circ}$ and $45^{\circ}$ before maximum elongation. Because of the low opening angle of the rings system, the reference zone is slightly contaminated by the A ring beyond $25^{\circ}$ from maximum elongation. However we have checked that all profiles do not present systematic deviations in the reference zone after this operation.

\subsubsection{Subtraction of the $F$ ring background and correction of illumination effects}

A synthetic median profile (SMP) of the F ring is built by taking the median value of all profiles at each longitude relative to elongation. The SMP is a template of the
F ring. It is subtracted from all profiles in order to reveal local brightness variations.

The apparent brightness of the $\mathrm{F}$ ring changes along its path around the ansa due to geometrical effects (the length of a ring segment included in one pixel depends on longitude) and probably to shadowing by the A ring (N96). Indeed the F ring brightness decreases abruptly after maximum elongation on the west ansa, as noticed in N96. To correct for these effects and maintain embedded features at a constant level of brightness, all profiles are divided by the SMP. Taking into account the subtraction of the F ring background, all profiles are processed according to the formula: Final Profile $=($ profile$-\mathrm{SMP}) / \mathrm{SMP}$. Dividing by the SMP increases noise in dim regions. This method preserves the brightness of objects within $20 \%$ before and after their passage through the maximum elongation (as observed on arcs $\mathrm{B}, \mathrm{C}$ and on the $10^{\circ}$ arc of N96, see below) and allows tracking of objects.

\subsubsection{Construction of the longitudinal F ring profile}

Each (final) profile is precessed to the standard epoch of November 21.5 TDT (terrestrial dynamic time) at Saturn, with a mean orbital motion of $582.05^{\circ} /$ day (N96). This allows direct comparison with N96 results. Finally a mean longitudinal profile is computed by averaging all precessed profiles.

The resulting CFHT and HST profiles are presented in Fig. 2. The derived HST full azimuthal profile (see Fig. 2c) is in close agreement with the one published in N96, in terms of the positions of objects and relative brightnesses, although our profile may appear somewhat noisier than the one published in N96. In this profile, the correction for the distortion of the $\mathrm{WF} / \mathrm{PC} 2$ camera has not been considered, resulting in an azimuthal error less than $\sim 0.6^{\circ}$. The vertical axis is scaled such that the maximum intensity of Pandora, as observed in the processed profiles, equals 1 .

\section{Results}

In this section the $\mathrm{F}$ ring azimuthal profile obtained from CFHT observations is described and compared with the HST profile. Orbital solutions for four detected structures are then calculated.

\subsection{F ring longitudinal profile and new objects}

\subsubsection{CFHT images}

The complete azimuthal coverage of the data set ranges between $50^{\circ}$ to $230^{\circ}$. But the region extending from $50^{\circ}$ to $80^{\circ}$ has been covered by only a few suitable images $(\sim 10)$ and is deeply embedded in the very strong scattered light of Saturn's globe. Apart from Pandora appearing at $62^{\circ}$, nothing is detected below $80^{\circ}$. Janus contaminates CFHT profiles around $210^{\circ}$ longitude. The region of interest then extends between $80^{\circ}$ and $200^{\circ}$ (Fig. 2a). In this range, 


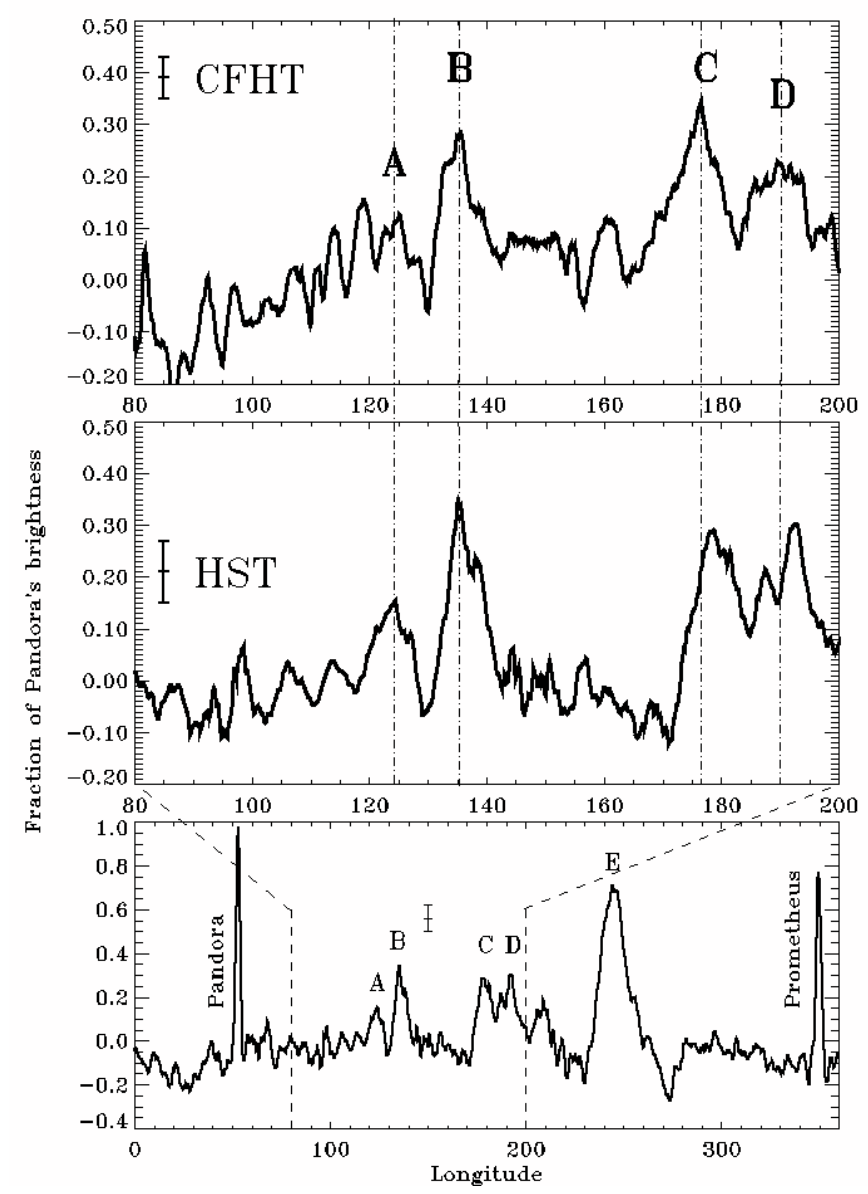

Fig. 2. Longitudinal brightness profiles extracted from 160 CFHT images of the west ansa and 20 HST images of both ansae (a) and b) respectively) in the common longitudinal range. Figure 1c is the full HST profile with complete azimuthal coverage, obtained with our data processing. Error bars are $\pm 1 \sigma$. Negative values mean that brightness is locally lower than the background of the ring. All profiles are precessed to the standard epoch 1995 November 21.5 TDT at Saturn. The B and $\mathrm{E}$ arc are refered by N96 as the " $7^{\circ}$ arc" and the " $10^{\circ}$ arc" respectively. The orbit of the $\mathrm{E}$ arc has been determined by P2000

the average error bar $(1 \sigma)$ is about $4 \%$ of Pandora's brightness.

Two structures (B and $\mathrm{C}$ ) are detected with more than $5 \sigma$ confidence level, with mean central longitudes of $135^{\circ}$ and $177^{\circ}$ respectively. The B object is the " $7^{\circ}$ arc" in N96. Their maximum brightness is $30 \%$ to $40 \%$ of Pandora's. Compared to the $3^{\circ}$-azimuthal extension of a point-like source due to the PSF (as observed for Pandora), it is clear that $\mathrm{B}$ and $\mathrm{C}$ are azimuthally extended objects. The B object has a $F W H M$ of $\sim 7^{\circ}$. It is observed before (from 5h10 to $6 \mathrm{~h} 30 \mathrm{UT}$ ) and after its passage through maximum elongation (from $7 \mathrm{~h} 40$ to $8 \mathrm{~h} 45 \mathrm{UT}$ ). Feature $\mathrm{C}$ has a $F W H M$ of $10^{\circ}$. It is detected during $20 \mathrm{~min}$ before maximum elongation (from $5 \mathrm{~h} 10$ to $5 \mathrm{~h} 30$ ) and during $80 \mathrm{mn}$ after maximum elongation (from 5 h50 to 7 h10 UT).

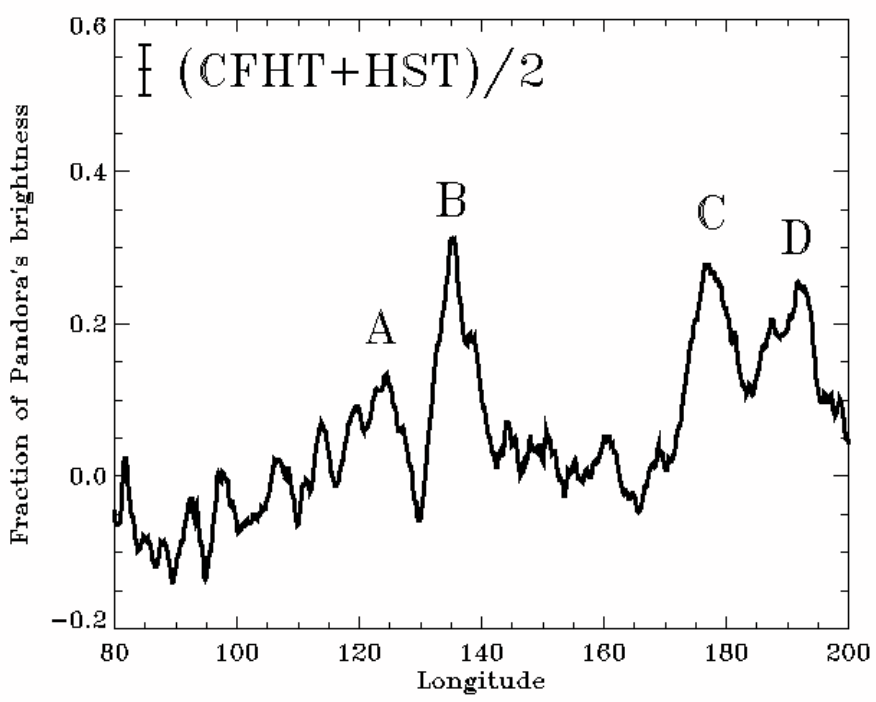

Fig. 3. Mean of CFHT and HST profiles presented in Figs. 2a and $2 \mathrm{~b}$ in the common azimuthal region at the standard epoch. Error bar is $\pm 1 \sigma$

A few other bumps also seem to appear with lower confidence level $(\sim 2-3 \sigma)$ below $120^{\circ}$ and at $124^{\circ}, 163^{\circ}$ and $190^{\circ}$. Only two of them may be tracked on many images with a keplerian motion. The A object, located at $124^{\circ}$, is nearly marginal here and seems to extend from $117^{\circ}$ to $129^{\circ}$ and has an intensity about $15 \%$ of Pandora's. It is detected from $5 \mathrm{~h} 50$ to $6 \mathrm{~h} 40$ UT ( $\sim 30$ images). The D object (located at $190^{\circ}$ ) may be followed from $5 \mathrm{~h} 40$ to $6 \mathrm{~h} 20$ UT. Its azimuthal shape is uncertain because of numerous nearby artifacts on the images where it is detected.

\subsubsection{Comparison with HST data}

Comparison of CFHT and HST profiles indicates that at least structures B and C lasted over a 24 to 36 hours time interval. Indeed both profiles are in good agreement within $1 \sigma$ (see Figs. 2a and $2 \mathrm{~b}$ ). Because of the small number of images, the noise varies significantly with longitude in the HST profile, depending on the number of images covering each region. It is on average $\sim 6 \%$ of Pandora's brightness. The azimuthal extension of a point-like object is also $3^{\circ}$ (measured on Pandora and Prometheus), but here it is mainly due to smearing. Objects $\mathrm{B}$ and $\mathrm{C}$ are present in both profiles. The shape and position of object $\mathrm{B}$ fits particularly well. C appears slightly narrower in the HST profile, but both are compatible within $1 \sigma$ in intensity. Its $F W H M$ is about $9^{\circ}$ in the HST profile.

A better signal to noise ratio is simply obtained by averaging CFHT and HST profiles (see Fig. 3). The new error bar at $1 \sigma$ is $3.3 \%$ of Pandora's brightness, which improves by factors 1.2 and 1.8 upon the signal to noise ratio of CFHT and HST profiles respectively. In the averaged profile the confidence level of objects $\mathrm{B}$ and $\mathrm{C}$ increases to $9 \sigma$ and $8 \sigma$ respectively. Objects $\mathrm{A}$ and $\mathrm{D}$, that were 
marginal in the CFHT profile, appear now at $5.5 \sigma$ and $5 \sigma$ respectively, showing these are real structures. Whereas the shape of A is in good agreement between CFHT and HST profiles, the shape of D is more peaked in the HST profile, and is centered at $192^{\circ}$. Features below $120^{\circ}$ and at $164^{\circ}$ are comparable to the error bar in the averaged profile. The simultaneous presence of structures A, B, C, $\mathrm{D}$ with same positions and similar shapes in both CFHT and HST data confirms that these are real and extended objects. They are designated as "arcs" hereafter.

\subsection{Orbital fit}

The computation of orbital solutions has been performed using moments J2 to J4 of Saturn's potential (Campbell \& Anderson 1989). The location of objects A, B, C and D are measured on the profiles where the Saturn's scattered light and the template of the F ring (the SMP) has been subtracted. The center is determined "by eye" by comparing at least three successive profiles where a given object appears clearly. Positions are then converted into distance from the center of Saturn projected onto the planet's equatorial axis. For HST data, profiles in which objects are measured are corrected for the distortion of the PC camera. Only the best points were considered with the longest time baseline, as it is the most important factor for an accurate orbit determination. The CFHT data points are presented in Table 1, in which the filter as well as the current longitude of each object are also reported.

Semi-major axis and longitude at epoch are determined by a least square fit of the position of objects projected onto the equatorial axis of Saturn. Error bars are obtained by introducing a random noise in the input positions, with dispersion equals to the spatial uncertainty (0.09" for CFHT and $0.035^{\prime \prime}$ for HST).

A circular fit was performed first. The results are presented in Table 2. They confirm that arcs A to D are located between Pandora and Prometheus $(\sim 140050 \mathrm{~km})$. Since such elongated structures are expected to be embedded in the $\mathrm{F}$ ring, we also performed a non-circular fit assuming the eccentricity and longitude of pericenter of the F ring, but the residuals did not significantly decrease.

If it is assumed that objects A, B, C, D are embedded in the $\mathrm{F}$ ring, an estimate of its semi-major axis in 1995 is calculated by taking the mean semi-major axis. Based on the circular fit we obtain $\langle a\rangle=140060$ $\pm 60 \mathrm{~km}\left(\Leftrightarrow n=582.9 \pm 0.4^{\circ} /\right.$ day $)$, with a physical width of $\pm 20 \mathrm{~km}$ around the mean, calculated by taking the standard deviation of the four derived semi-major axes. For non-circular fit we obtain $\left\langle a_{\mathrm{ex}}\right\rangle=140050 \pm 60 \mathrm{~km}$ ( $\Leftrightarrow n=583.0 \pm 0.4^{\circ} /$ day), with a physical width of $\pm 30 \mathrm{~km}$ around the mean. Combining both data set allows to significantly improve the orbital solution of arc B $\left(584 \pm 4^{\circ} /\right.$ day) as reported in N96. This gives also the first determination of the orbits of $\mathrm{A}, \mathrm{C}$ and $\mathrm{D}$ arcs.

\section{Discussion}

\subsection{Comparison with August and November 1995 observations}

Another $10^{\circ}$-long arc has been seen by N96 (it is designed here as the "E arc", see Fig. 2c) on November 21st 1995. Its orbit has been determined by P2000 using November 17th, 18th and 21st HST images. Their value of $140074 \pm$ $30 \mathrm{~km}$ is consistent $(1 \sigma)$ with the $\mathrm{A}, \mathrm{B}, \mathrm{C}$ and $\mathrm{D}$ arcs location.

Some other objects discovered in August 1995, during the Earth crossing seemed also to be close to and somewhat below $140000 \mathrm{~km}$ (Roddier et al. 1996a, N96; Ferrari et al. 1997, P2000). However most of them appeared to be point-like, and thus possibly of a different nature than features A to E. Based on N96 orbital solutions, S6 and S7 are located at $139900 \mathrm{~km}$ and $139100 \mathrm{~km}$ (with error bars of $70 \mathrm{~km}$ and $260 \mathrm{~km}$ respectively). The orbit of S5 is still a matter of debate (Nicholson, private communication). Its semi-major was initially estimated as $139860 \pm 130 \mathrm{~km}$ by N96 and $140050 \pm 100 \mathrm{~km}$ by Roddier et al. (1996a), but two recent studies, combining different data sets, gives uncompatible results: P2000 find 140208 $\pm 50 \mathrm{~km}$ and McGhee et al. (submitted to Icarus) report $a=139690 \pm 90 \mathrm{~km}$. A few other objects (S11 to S19) where also discovered by Roddier et al. (1996a, 1996b). Their estimated orbits are not accurate enough to give us more information on the $\mathrm{F}$ ring environment (error bars are $\pm 1000 \mathrm{~km}$ and $\pm 2000 \mathrm{~km}$ for $\mathrm{S} 8$ and $\mathrm{S} 9$, and $\pm 500 \mathrm{~km}$ for objects S11 to S15).

\subsection{Comparison with 1980-81 Voyager observations}

Values of $<a>$ and $<a_{\mathrm{ex}}>$ are however quite far $(3 \sigma)$ from all estimates of the $\mathrm{F}$ ring radius derived from Voyager images, by about $-150 \mathrm{~km}$. Indeed Synnott et al. (1983) gives $a=140185 \pm 30 \mathrm{~km}$. A recent study of Voyager images (Ferrari et al. 1997, 1998) reveals that, at the Voyager epoch, some of the brightest structures of the $\mathrm{F}$ ring move on different orbits, with a measured dispersion of $95 \pm 15 \mathrm{~km}(1 \sigma)$. The measured semi-major axis is $140219 \pm 19 \mathrm{~km}$ derived from Voyager 1 data and $140205 \pm 10 \mathrm{~km}$ derived from Voyager 2 data. All those results are consistent with French and Nicholson who used a different approach based on Voyager and stellar occultation data (unpublished work of French \& Nicholson, reported in N96) giving $a=140209 \pm 4 \mathrm{~km}$.

\subsection{F ring radial structure}

The width of the F ring in Voyager images is up to $300 \mathrm{~km}$ and the ring is formed of four eccentric strands (Murray et al. 1997), $\mathrm{F} \alpha, \mathrm{F} \beta, \mathrm{F} \gamma$ and $\mathrm{F} \delta$, which radial width is $50 \mathrm{~km}$ in average and with respective semi-major axes $140089 \mathrm{~km}, 140176 \mathrm{~km}, 140219 \mathrm{~km}$ and $140366 \mathrm{~km}$. The $\mathrm{F} \gamma$ strand is by far the brightest and may be the "core" 
Table 1. CFHT data points of arcs A, B, C, D upon which orbital solutions were determined. The distance (in km) is measured from Saturn's center, projected perpendicularly on the equatorial axis of the west ansa. The longitude is calculated on the basis of orbital solutions given in Table 2. It is measured from the ascending node of Saturn's equatorial plane on the Earth's equator at the standard epoch

\begin{tabular}{lllll}
\hline Object & $\begin{array}{l}\text { Decimal day (UTC) } \\
\text { of } 1995 \text { November }\end{array}$ & $\begin{array}{l}\text { Distance }(\mathrm{km}) \text { on } \\
\text { equatorial axis }\end{array}$ & $\begin{array}{l}\text { Longitude } \\
\text { (degrees) }\end{array}$ & Filter \\
\hline A & 20.283900 & 125847 & 103.7 & $K$ \\
A & 20.289005 & 127699 & 105.5 & $K$ \\
A & 20.293935 & 131403 & 109.5 & $K$ \\
A & 20.295486 & 132560 & 110.9 & $K$ \\
A & 20.300613 & 134875 & 114.1 & $K$ \\
A & 20.301250 & 135570 & 115.2 & $K$ \\
A & 20.351597 & 135583 & 144.3 & $K$ \\
& & & & \\
B & 20.241563 & 111493 & 92.5 & $H$ \\
B & 20.243727 & 113345 & 93.8 & $H$ \\
B & 20.263044 & 126541 & 104.3 & $J$ \\
B & 20.266250 & 127930 & 105.7 & $J$ \\
B & 20.266944 & 128856 & 106.7 & $J$ \\
B & 20.270891 & 130940 & 108.9 & $J$ \\
B & 20.277755 & 134412 & 113.4 & $J$ \\
B & 20.337269 & 133949 & 146.8 & $K$ \\
B & 20.338669 & 133023 & 148.0 & $K$ \\
B & 20.343287 & 132097 & 149.2 & $K$ \\
& & & & \\
C & 20.214919 & 136959 & 117.7 & $K$ \\
C & 20.269444 & 132560 & 148.6 & $J$ \\
C & 20.270359 & 131403 & 150.0 & $J$ \\
C & 20.272118 & 130245 & 151.3 & $J$ \\
C & 20.273333 & 129319 & 152.3 & $J$ \\
C & 20.277755 & 126541 & 155.1 & $J$ \\
C & 20.288356 & 120753 & 160.2 & $K$ \\
C & 20.296134 & 114503 & 164.9 & $K$ \\
C & 20.315706 & 97139 & 165.8 & $K$ \\
D & 20.248773 & 128625 & 162.9 & $J$ \\
D & 20.265012 & 117744 & $J$ \\
D & 20.266250 & 117281 & $J$ \\
D & 20.267894 & 115429 & & $J$ \\
D & 20.270891 & 114503 & & $J$ \\
D & 20.278275 & & 169.9 & $J$ \\
\hline & & & & $J$ \\
\hline
\end{tabular}

of the F ring with centimeter-sized bodies (Showalter et al. 1992; Murray et al. 1997), in which the brightest features (like clumps or arcs) were detected at the Voyager epoch (Ferrari et al. 1997; Showalter 1997). The five arcs seen in November 1995 (A, B, C, D and E) seem to be gathered around $140060 \mathrm{~km}$, close to the the faint $\mathrm{F} \alpha$ strand. Consequently, if the 1995 November arcs belong to a F ring core, this may imply a radial restructuring or a spreading of the ring in the meantime.

\subsection{Possible implications}

We suggest below, as a direction for future works, that some close encounter with one of the shepherding satellites may have reshaped the $\mathrm{F}$ ring in the last twenty years. Such an hypothesis may not be excluded since the dynamical evolution of this ring is still a puzzle for the scientific community. The evolution of the $\mathrm{F}$ ring may critically depend on its two shepherding satellites, Pandora and Prometheus (see for example: Dermott 1981; Showalter \& Burns 1982; Lissauer \& Peale 1986). During the 1995 crossings, Prometheus was lagging its expected position by $\sim 19^{\circ}$ (N96). It has been suggested that an encounter of the $\mathrm{F}$ ring with Prometheus may have happened in between 1991 and 1994 (Murray \& Giuliatti Winter 1996) due to the precessional variation of the orbits owing to Saturn's oblateness. The dynamical consequence of this encounter on the structure of the F ring is not currently known. It might result in breaking of the strands and in the creation of structures in the inner regions of 
Table 2. Orbital fit for A, B, C, D derived by combining HST and CFHT data. The orbital fit for Pandora is also reported as it is detected in both HST and CFHT data (below $80^{\circ}$ ). The standard epoch is 1995 November 21.5 TDT at Saturn. Longitude is measured from the ascending node of Saturn's equatorial plane on the Earth's equator at the standard epoch. 0: Circular orbit. 1: Eccentricity and longitude of pericenter of the $\mathrm{F}$ ring at the standard epoch are assumed to be $e=0.0029$ and $\omega=292.5^{\circ}$ (Synnott et al. 1983). 2: Eccentricity and longitude of pericenter of Pandora are assumed to be $e=0.0044$ and $\omega=274.7^{\circ}$

\begin{tabular}{lllll}
\hline Object & $\begin{array}{l}\text { Semi-major axis } \\
(\mathrm{km})\end{array}$ & $\begin{array}{l}\text { Longitude at epoch } \\
(\text { degrees })\end{array}$ & $\begin{array}{l}\text { Residuals } \\
(\mathrm{km})\end{array}$ & $\begin{array}{l}\text { Mean Motion } \\
(\mathrm{deg} / \mathrm{day})\end{array}$ \\
\hline $\mathrm{A}^{0}$ & $140070 \pm 166$ & $122.85^{\circ} \pm 1.3$ & 751 & $582.92 \pm 0.96$ \\
$\mathrm{~B}^{0}$ & $140083 \pm 108$ & $135.81^{\circ} \pm 0.6$ & 617 & $582.80 \pm 0.60$ \\
$\mathrm{C}^{0}$ & $140050 \pm 119$ & $177.32^{\circ} \pm 0.8$ & 643 & $583.05 \pm 0.68$ \\
$\mathrm{D}^{0}$ & $140046 \pm 141$ & $192.47^{\circ} \pm 0.9$ & 691 & $583.07 \pm 0.91$ \\
& & & & \\
$\mathrm{~A}^{1}$ & $140075 \pm 158$ & $122.66^{\circ} \pm 1.2$ & 723 & $582.89 \pm 1.02$ \\
$\mathrm{~B}^{1}$ & $140081 \pm 100$ & $135.43^{\circ} \pm 0.6$ & 598 & $582.85 \pm 0.61$ \\
$\mathrm{C}^{1}$ & $140017 \pm 109$ & $177.66^{\circ} \pm 0.5$ & 611 & $583.23 \pm 0.68$ \\
$\mathrm{D}^{1}$ & $140021 \pm 139$ & $192.69^{\circ} \pm 1.0$ & 657 & $583.23 \pm 0.87$ \\
Pandora $^{2}$ & $141792 \pm 87$ & $52.0^{\circ} \pm 0.3$ & 512 & $572.30 \pm 0.53$ \\
\hline
\end{tabular}

the $\mathrm{F}$ ring. Indeed a massive body is able to scatter its environment inside three Hill's radii (Nishida 1983; Ida \& Makino 1993) that is about $300 \mathrm{~km}$ for Prometheus (assuming a mass ratio of $\sim 10^{-9}$ between Prometheus and Saturn). Then in case of an instantaneous close approach to the F ring, estimated at $\sim 50 \mathrm{~km}$ (Murray \& Giuliatti Winter 1996), Prometheus might be able to perturb the ring over a distance of $300 \mathrm{~km}$, i.e. about the full width of the F ring. Such a model has been recently considered (abstract of Showalter et al. 1999; Nicholson, private communicaton), and first results show that Prometheus may perturb a portion of the $\mathrm{F}$ ring after each close encounter. However the resulting radial displacement seems to be about 1 kilometer only.

In addition, during the interaction, Prometheus at its apocenter is close to the $\mathrm{F}$ ring's pericenter, yielding strong relative velocities of $\sim 30 \mathrm{~m} / \mathrm{s}$. Such high-velocity encounters may result in catastrophic disruption of bodies initially in the $\mathrm{F}$ ring. The inner region of the $\mathrm{F}$ ring may be consequently populated with fragments especially if a belt of kilometer-sized moonlets exists between Prometheus and Pandora, as originally suggested by Cuzzi \& Burns (1988).

\section{Conclusion}

We have reported observations of the crossing of Saturn's ring-plane by the Sun, on 1995 November 20th, made with the $3.6 \mathrm{~m}$ CFH Telescope, using the UHAO adaptive optics system. Four azimuthally extended structures (A, B, C , D) have been detected, with central longitude at epoch of $123^{\circ}, 136^{\circ}, 177^{\circ}$ and $193^{\circ}$ (see Table 2), on 1995 November 21.5 TDT at Saturn. These structures have been also seen one day later in HST images (N96). The combination of both CFHT and HST data sets provides an accurate estimate of their orbit. An orbital fit locates them between $140020 \mathrm{~km}$ and $140080 \mathrm{~km}$, with error bars of $120 \mathrm{~km}$ on average. The mean circular orbit is $140060 \pm$ $60 \mathrm{~km}$, that is consistent with the orbit of another bright arc observed at the same epoch (P2000). However, this represents a significant discrepancy $(3 \sigma)$ with previous estimates of the F ring's radius derived from Voyager images (located at $140200 \mathrm{~km}$ on average). If the five arcs observed in November 1995 belong to a F ring core, this would imply a radial restructuring or a spreading of the $\mathrm{F}$ ring between 1980 and 1995, whose explanation has still to be found. In order to understand the F ring's dynamics, better spatial resolution and longer time baselines observations are required. The Cassini encounter in 2004 will probably be a major step in the understanding of the $\mathrm{F}$ ring mysteries.

Acknowledgements. The authors thank P. D. Nicholson for his helpful comments, as a referee.

\section{References}

Bosh, A. S., \& Rivkin, A. S. 1996, Sci, 272, 518

Cuzzi, J. N., \& Burns, J. A. 1988, Icarus, 74, 284

Campbell, J. K., \& Anderson, J. D. 1989, AJ, 97, 1485

Dermott, S. F. 1981, Nat, 290, 454

Ferrari, C., Brahic, A., Charnoz, S., Thébault, P., \& Roddier, F. 1997, BAAS 29, 998

Ferrari, C., Brahic, A., Charnoz, S., \& Thébault, P. 1998, Actes du deuxième colloque national de planétologie de l'INSU, vol. $2,57.5$

Holtzman, J. A., Burrows, C. J., Casertano, S., et al. 1995, PASP, 107, 156

Ida, S., \& Makino, J. 1993, Icarus, 106, 210

Lissauer, J. J., \& Peale, S. J. 1986, Icarus, 67, 358

Murray, C. D, \& Giuliatti Winter, S. M. 1996, Nat, 380, 139

Murray, C. D., Gordon, M. K., \& Giuliatti Winter, S. M. 1997, Icarus, 129, 304

Nicholson, P. D., Showalter, M. R., Dones, L., et al. 1996, Sci, 272,509

Nishida, S. 1983, Prog. Theor. Phys., 70, 93 
Poulet, F., Sicardy, B., Nicholson, P. D., Karkoschka, E., \& Caldwell, J. 2000, Icarus, 144, 135

Porco, C., Danielson, G. E., Goldreich, P., Holberg, J. B., \& Lane, A. L. 1984, Icarus, 60, 17

Roddier, C., Roddier, F., Brahic, A., et al. 1996a, IAU Circ. 6407

Roddier, C., Roddier, F., Brahic, A., et al. 1996b, IAU Circ. 6515

Roddier, F., Roddier, C., Brahic, A., Dumas, C., et al. 2000, Icarus, 143, 299
Showalter, M. R. \& Burns, J. A. 1982, Icarus, 52, 526

Showalter, M. R., Pollack, J. B., Ockert, M. E., Doyle, L. R., \& Dalton, J. B. 1992, Icarus, 100, 394

Showalter, M. R. 1997, BAAS, 29, 999

Showalter, M. R., Dones, L., \& Lissauer, J. J. 1999, BAAS, 31, 1141

Sicardy, B., Poulet, F., Beuzit, J.-L., \& Prado, P. 1996, IAU Circ. 6395

Synnott, S. P., Terrile, R. J., Jacobson, R. A., \& Smith, B. A. 1983, Icarus 53, 156 